1 Hacettepe Journal of Mathematics and Statistics

h Volume 48 (1) (2019), 274-289

\title{
Estimation of the waiting time of patients in a hospital with simple Markovian model using order statistics
}

\author{
Soma Dhar*, Lipi B. Mahanta ${ }^{\dagger \ddagger}$ and Kishore K. Das ${ }^{\S}$
}

\begin{abstract}
In this paper, consider a single server queue in a hospital environment whose service time is governed by a Markov process. It is possible that the server changes its service speed many times while serving a patient. Here we have studied the order statistics for waiting time distribution where the probability density function of single order statistics $\phi_{i: n}, \mathrm{cu}-$ mulative density function of $\Phi_{i: n}$, joint probability density function of $\phi_{i: n}$ and $\phi_{j: n}$, probability density function of extreme order statistics. Also have been considered the moments and recurrence relation of order statistics, the probability density function of sample range and sample median. We derive minimum and maximum order statistics of the service time of patients in the system using first step analysis to obtain an insight on the service process. Further, we use order statistics to compute performance measures such as average queue length and waiting time for severe diseases especially in the outpatient department. This result effectively establishes that as the number of server increases, then the utmost and the minimum waiting time of the patients decreases. Also illustrate the application of the simple Markovian model by using real hospital data.
\end{abstract}

Keywords: Waiting time, Service time, Patients, Order statistics.

2000 AMS Classification: 60K25

Received : 11.09.2017 Accepted : 28.06.2018 Doi : 10.15672/HJMS.2018.607

*Department of Statistics, Gauhati University, Guwahati., Email: somadhar7@gmail.com

$\dagger$ Centre for Computational and Numerical Sciences, Institute of Advanced Study in Science and Technology, Guwahati - 781035, India Email: Ibmahanta@iasst.gov.in

$\ddagger$ Corresponding Author.

$\S$ Department of Statistics, Gauhati University, Guwahati, Email: daskkishore@gmail.com 


\section{Introduction}

The theory of queues has wide applications in the field of health-care management system. The study of queuing systems in hospitals has often been concerned with the busy period and waiting time, because they play a very significant role there. A queuing system is normally described by the patient's entry into a queue, who are then served at a service point by the server (doctor), after which they leave the queue.

Dhar et al.[9] studied the comparison between single and multiple Markovian queuing model in an outpatient department. Also Mahanta et al.[13] proposed a single server queueing model for severe diseases especially in outpatient department. Further consider the infinite server queues with time-varying arrival and departure pattern when the parameters are varying with time derive by Dhar et al.[16].

Order statistics are widely used in applications of statistical models and inference. Both describes random variables which are arranged in order of magnitude. According to Aleem [1], usually the ordered values of independent and identically distributed samples arranged in ascending order of magnitude are known as order statistics. The simplest and most important function of order statistics is the sample cumulative distribution function $F_{n}(x)$. Suppose $X_{1}, X_{2}, \ldots, X_{n}$ are $n$ jointly distributed random variables. Arranging the X's in increasing order of magnitude, $X_{1: n}, X_{2: n}, \ldots, X_{n: n}$ are said to be smallest, second smallest and largest order statistics. Thus $X_{1: n}<X_{2: n}, \ldots,<X_{n: n}$. Arnold et al. [2] and David and Nagaraja [7] studied order statistics and functions of these statistics as it plays an important role in wide range of theoretical and practical problems such as characterizations of probability distributions and goodness of fit test, entropy estimation, analysis of censored samples, reliability analysis, quality control and strength of materials. Order statistics arise naturally in many real-life applications involving data relating to life testing studies proved by Shawky[10]. Aleem [1] reported that methods of interpretation based on order statistics are most efficient and are used extensively because of robustness and parsimonious nature. The sample mean and standard deviation provide efficient estimators of the corresponding population parameter under the assumption of normality, but sample range is simpler to use than the sample standard deviation in statistical quality control and the sample median and its deviation furnish more robust estimators when the population have long tail. Extreme (largest and smallest) values statistics, which is an offspring of order statistics, has its importance in hydrology, aeronautics, oceanography, material strength, signal processing and meteorology. Moments of order statistics also plays an important role in the area of quality control testing and reliability. According to David and Nagaraja [8] moments of order statistics can be used to measure the failure rate of reliability and to predict the failure of future events.

A recursive procedure for computing the moments of the busy period for the singleserver model can be found in Tarabia [12]. Limit theorems are proved by investigating the extreme values of the maximum queue length, the waiting time and virtual waiting time for different queue models in literature. Serfozo [14] discussed the asymptotic behavior of the maximum value of birth-death processes over large time intervals. Serfozo's results concerned the transient and recurrent birth-death processes and related $M / M / c$ queues. Asmuseen[4] introduced a survey of the present state of extreme value theory for queues and focused on the regenerative properties of queuing systems, which reduced the problem to study the tail of the maximum of the queuing process $X(t)$ during a regenerative cycle, where $X(t)$ is in discrete or continuous time. Artalejo et al.[3] presented an efficient algorithm for computing the distribution for the maximum number of 
customers in orbit and in the system during a busy period for the $M / M / c$ retrial queue. The main idea of their algorithm is to reduce the computation of the distribution of the maximum customer number in orbit by computing certain absorption probabilities. For more details of extreme value in queues by Park [15].

In this paper, we studied the maximum and minimum service and waiting time respectively, of the patients who suffer from severe disease especially in public hospital. Here we considered one of the leading public hospitals of the region, viz. Pandu P.H.C/F.R.U, Guwahati where it was observed that there was a heavy flow of patients throughout the day. Data was collected from Hospital (viz. outpatient department) and from other allied sources. The current chapter will have utility for various practical problems for which the distributions of order statistics play a role and the queuing theory implicit to the health related problems.

\section{Formulation of the problem}

Let $X_{1}, X_{2}, \ldots, X_{n}$ be a random sample from a continuous population with probability density function $\phi(x)$ and cumulative distribution function $\Phi(x)$ and $X_{1: n}, X_{2: n}, \ldots, X_{n: n}$ be the order statistics obtained by arranging the random sample in increasing order of magnitude. Then according to David and Nagaraja [7] the probability density function of the $i^{t h}$ order statistics $X_{i: n}, 1<i<n$ is given by

$$
\phi_{i: n}(x)=\frac{n !}{(i-1) !(n-i) !}[\Phi(x)]^{i-1}[1-\Phi(x)]^{n-i} f(x),-\infty<x<\infty
$$

The probability density functions of smallest and largest order statistics are given by Arnold et al.[2] as

$$
\phi_{1: n}=n[1-\Phi(x)]^{n-1} \phi(x),-\infty<x<\infty
$$

and

$$
\phi_{n: n}=n[\Phi(x)]^{n-1} \phi(x),-\infty<x<\infty
$$

respectively. According to Arnold et al.[2] the cumulative density functions of smallest and largest order statistics are given as

$$
\Phi_{1: n}=1-[1-\Phi(x)]^{n},-\infty<x<\infty
$$

and

$$
\Phi_{n: n}=[\Phi(x)]^{n},-\infty<x<\infty
$$

respectively.

The $p^{t h}$ order moment for the $i^{t h}$ order statistics is also given by Arnold et al.[2] as

$$
\begin{aligned}
\mu^{\prime}(r: n)= & \int_{-\infty}^{\infty} x^{p} \phi_{r: n}(x) d x \\
= & \frac{n !}{(i-1) !(n-i) !} \int_{-\infty}^{\infty} x^{p}[\Phi(x)]^{i-1}[1-\Phi(x)]^{n-i} \\
& \phi(x) d x,-\infty<x<\infty
\end{aligned}
$$

Assuming $u=X_{i: n}$ and $v=X_{j: n}$ as the $i^{\text {th }}$ and $j^{\text {th }}$ order statistics, $1<i<j<n$ from $n$ independent random variable each with probability density function $\phi(x)$, the joint density function of $u=X_{i: n}$ and $v=X_{j: n}$ is given by Arnold at el. [2], as

$$
\begin{aligned}
\phi(u, v: n)= & c^{\prime}(i, j, n)[\Phi(u)]^{i-1}[\Phi(v)-\Phi(u)]^{i-j-1}[1-\Phi(v)]^{n-j} \\
& \phi(v) \phi(u),-\infty<u<v<\infty
\end{aligned}
$$


where $c^{\prime}(i, j, n)=\frac{n !}{(i-1) !(i-j-1) !(n-i) !}$

David and Nagaraja [7], has given the probability density function of double moment as

$$
\begin{aligned}
\mu_{p, q}^{\prime}(i, j: n)= & \frac{n !}{(i-1) !(i-j-1)(n-j) !} \\
& \iint_{0<u<v<\infty} u^{p} v^{q} d u d v,-\infty<x<\infty
\end{aligned}
$$

Arnold at el.[2] defined the sample range as $W_{n}=X_{n: n}-X_{1: n}$ and

$$
\begin{aligned}
\phi_{w_{n}}(w)= & n(n-1) \\
& \int_{-\infty}^{\infty}\left[\Phi\left(x_{1}+w\right)-\Phi\left(x_{1}\right)\right]^{n-2} \phi\left(x_{1}\right) \phi\left(x_{1}+w\right) d x_{1}, 0<w<\infty
\end{aligned}
$$

Percentage points of distributions are the most fundamental tools used in test of hypothesis to take decision about various situations of the population based on sample observations and also used to express the difference of risks of probabilities. The percentile points are the point on the measurement scale below which a specified percentage of score falls. In many applications involving these distributions percentage points are required. Bagui[5] defined the percentage points depends on the evaluation of the inverse probability function. In general, percentile points of the distributions have been obtained using approximation, interpolation formula, quadrature formula and by simulation. According to White [11] the $p^{\text {th }}$ percentile equation of distribution is given as

$$
\int_{0}^{\chi} \phi(x) d x=p
$$

where $p$ denotes level of significance.

The $p^{t h}$ percentile equation of smallest and largest order statistics are given as

$$
\Phi_{1: n}=\int_{0}^{\chi} \Phi_{1: n} d x=1-[1-\Phi(x)]^{n}=p
$$

and

$$
\Phi_{n: n}=\int_{0}^{\chi} \Phi_{n: n} d x=[\Phi(x)]^{n}=p
$$

Let $X_{1: n}, X_{2: n}, \ldots, X_{n: n}$ be the order statistics of a random variables $X_{1}, X_{2}, \ldots, X_{n}$. Also let $T_{i: n}=X_{i: n}-X_{i-1: n}, i=1,2,3, \ldots, n$, where $T_{i}$ represents the difference between each arrival into the system(inter-arrival) of the order statistics $X_{i: n}$ and $X_{i-1: n}$. Then the random variables $T_{1}, T_{2}, \ldots, T_{n}$ are called the inter-arrival time between the successive order statistics $X_{1: n}, X_{2: n}, \ldots, X_{n: n}$.

Here we consider the sample range $R$ which is denoted by

$$
R=\sum_{i=2}^{n} T_{i: n}
$$

Moreover, this can be used to construct the interval for the corresponding patients.

2.1. Theorem. Let $T_{1}, T_{2}, \ldots, T_{n}$ be the random sample of size $n$ from a continuous distribution with cumulative density function $\Phi$ and the probability density function $\phi$. Then the joint distribution of order statistics is given by

$$
\Phi_{T_{i}}(t)=1-\int_{-\infty}^{\infty}(i-1)\left(\begin{array}{c}
n \\
i-1
\end{array}\right) \Phi_{X}^{(i-2)}(y) 1-\Phi_{X}(x+t)^{(n-i+1)} \phi_{X}(x) d x
$$


Proof. We know that,

$$
\begin{aligned}
\Phi_{T_{i}}(t) & \left.=P\left(T_{i} \leq t\right)\right) \\
& =P\left(X_{(i)}-X_{(i-1)} \leq t\right) \\
& =P\left(X_{(i)} \leq X_{(i-1)}+t\right)
\end{aligned}
$$

Let $w$ be the region bounded by $X_{(i)} \leq X_{(i-1)}$ and $X_{(i)} \leq X_{(i-1)}+t$.

From equation (2.15),

$$
\begin{aligned}
\Phi_{T_{i}}(t)= & \iint_{w} \phi_{T_{i}, T_{i-1}}(x, y) d x d y \\
= & \int_{-\infty}^{\infty} \int_{x}^{x+t} \frac{n ! \phi_{X}(x) \phi_{X}(y)}{(i-2) !(n-i) !}\left[1-\Phi_{X}(y)\right]^{(n-i)} \Phi_{X}^{(i-2)}(y) \\
& {\left[\Phi_{X}(y)-\Phi_{X}(x)\right]^{i-i+1-1} d x d y } \\
= & \int_{-\infty}^{\infty} \frac{n ! \phi_{X}(x)}{(i-2) !(n-i) !} \Phi_{X}^{(i-2)}(y)\left(\int_{x}^{x+t} \phi_{X}(y)\left[1-\Phi_{X}(y)\right]^{(n-i)} d y\right) d x \\
= & \int_{-\infty}^{\infty} \frac{n ! \phi_{X}(x)}{(i-2) !(n-i) !} \Phi_{X}^{(i-2)}(y)\left|\frac{-\left[1-\Phi_{X}(y)\right]^{(n-i+1)}}{(n-i+1)}\right|_{x}^{x+t} d x \\
= & \int_{-\infty}^{\infty} \frac{n ! \phi_{X}(x)}{(i-2) !(n-i+1) !} \Phi_{X}^{(i-2)}(y) \\
& {\left.\left[-1-\Phi_{X}(x+t)^{(n-i+1)}+1-\Phi_{X}(x)\right]^{(n-i+1)}\right] d x } \\
= & \left.\int_{-\infty}^{\infty} \frac{n ! \phi_{X}(x)}{(i-2) !(n-i+1) !} \Phi_{X}^{(i-2)}(y) 1-\Phi_{X}(x)\right]^{(n-i+1)} d x \\
& -\int_{-\infty}^{\infty} \frac{n ! \phi_{X}(x)}{(i-2) !(n-i+1) !} \Phi_{X}^{(i-2)}(y) 1-\Phi_{X}(x+t)^{(n-i+1)} d x
\end{aligned}
$$

by using equation (2.15), since it is the integral of the $(i-1)^{t h}$ order statistics over $(-\infty, \infty)$

$$
\begin{aligned}
\Phi_{T_{i}}(t) & =1-\int_{-\infty}^{\infty} \frac{n ! \phi_{X}(x)}{(i-2) !(n-i+1) !} \Phi_{X}^{(i-2)}(y) 1-\Phi_{X}(x+t)^{(n-i+1)} d x \\
& =1-\int_{-\infty}^{\infty}(i-1)\left(\begin{array}{c}
n \\
i-1
\end{array}\right) \Phi_{X}^{(i-2)}(y) 1-\Phi_{X}(x+t)^{(n-i+1)} \phi_{X}(x) d x
\end{aligned}
$$

2.2. Corollary. Let $i=1$ and $i=n$ in Theorem 2.1

$$
\begin{aligned}
\Phi_{T_{1}}(t) & =P\left(T_{1} \leq t\right) \\
& =P\left(X_{(1)} \leq t\right) \\
& =1-[1-\Phi(t)]^{n}
\end{aligned}
$$

2.3. Theorem. Let $X_{(1)}, X_{(2)}, \ldots, X_{(n)}$ denote the order statistics of a random sample of size $n$ from a continuous distribution with probability density function $\phi_{X}(x)$ and a cumulative density function $\Phi_{X}(x)$. Then the probability density function of the $j^{\text {th }}$ order statistics is given by

$$
\phi_{X_{(j)}}(x)=\frac{n ! \phi_{X}(x)}{(j-1) !(n-j) !} \Phi_{X}^{(j-1)}(x) 1-\Phi_{X}(x)^{(n-j)}
$$

Proof. Proof of the theorem 2.3 given in Artalejo et al.[3]. 
2.4. Corollary. Let $\Phi_{T_{n}}(t)=X_{(n)}-X_{(n-1)}, 0<t<\infty$. Then

$P\left(T_{n} \leq t\right)=1-\int_{-\infty}^{\infty} n(n-1) \Phi_{X}^{(n-2)}(y) 1-\Phi_{X}(x+t) \phi_{X}(x) d x$

\section{Order statistics for waiting time distribution}

When a patient wait for service, the two most important characteristics that arises are (i) time spent in the queue and(ii) time spent in the system. Considering the system is in equilibrium, let $T_{q}$ and $T$ be the amount of time a customer spends in queue and in the system, respectively. However, the waiting time for service $\left(T_{q}\right)$ of an arriving customer is the amount of time required to serve the customers already in the system. The total time in system $T$ is $T_{q}+$ service time. When there are $\mathrm{n}$ customers in the system, since service times are exponential with parameter $\mu$, the total service time of $n$ customers is Erlang with probability density

$$
\phi_{n}(x)=e^{-\mu x} \frac{\mu^{n} x^{n-1}}{(n-1) !}
$$

Let $F q(t)=P(T q \leq t)$ be the distribution function of the waiting time $T_{q}$. Here $\Phi q(0)=P(T q=0)=P(Q=0)=1-\rho$. It is noted that because of the memoryless property of the exponential distribution, the remaining service time of the customer in service is also exponential with the same parameter $\mu$.

Let $d \Phi q(t)=P(t<T q \leq t+d t)$, for $t>0$, we have

$$
\begin{aligned}
d \Phi q(t) & =\sum_{n=1}^{\infty} p_{n} e^{-\mu t} \frac{\mu^{n} t^{n-1}}{(n-1) !} d t \\
& =(1-\rho) \sum_{n=1}^{\infty} \rho^{n} e^{-\mu t} \frac{\mu^{n} t^{n-1}}{(n-1) !} d t
\end{aligned}
$$

After simplification it is given by

$$
=\mu \rho(1-\rho) e^{-\mu(1-\rho) t} d t
$$

Because of the discontinuity at 0 in the distribution of $T_{q}$, we get

$$
\begin{aligned}
\Phi q(t) & =P(T q=0)+\int_{0}^{t} d \Phi q(t) \\
& =1-\rho e^{-\mu(1-\rho) t},
\end{aligned}
$$

The probability density function of the waiting time in the queue is given by Medhi [17]

$$
w_{q}(t)= \begin{cases}(1-\rho), & t=0 \\ \mu \rho(1-\rho) e^{-\mu(1-\rho) t}, & t>0\end{cases}
$$

The probability density function of the waiting time in the system is given by Bhat[6]

$$
w(x)=\mu(1-\rho) e^{-\mu(1-\rho) x}, \quad x \geq 0
$$

which is a exponential distribution with parameter $\mu(1-\rho)$

3.1. Derivation of $i^{\text {th }}$ order statistics for waiting time distribution. If $X_{1}, X_{2}$, $\ldots, X_{n}$ is a random sample from a continuous population with probability density function $\phi(x)$ and cumulative distribution function $\Phi(x)$ and $X_{1: n}, X_{2: n}, \ldots, X_{n: n}$ are the order statistics obtained by arranging the random sample in increasing order of magnitude, then the probability density function of the $i^{\text {th }}$ order statistics $X_{i: n}$ for waiting 
time distribution using (2.3), $1<i<n$ is given by

$$
\begin{aligned}
\phi_{i: n_{w}}(x)= & \frac{n !}{(i-1) !(n-i) !}\left[1-\rho e^{-\mu(1-\rho) x}\right]^{i-1} \\
& {\left[1-\left(1-\rho e^{-\mu(1-\rho) x}\right)\right]^{n-i} e^{-\mu x} \frac{\mu^{n} x^{n-1}}{(n-1) !} }
\end{aligned}
$$

Using binomial expression, the probability density function of $i^{\text {th }}$ order statistics $X_{i: n},(1<$ $i<n$ ) for waiting time distribution reduces to

$$
\begin{aligned}
\phi_{i: n_{w}}(x)= & \frac{n !}{(i-1) !(n-i) !} \sum_{r=0}^{i-1}\left(\begin{array}{c}
i-1 \\
r
\end{array}\right)(-1)^{r} \rho^{r} e^{-\mu(1-\rho)^{r}} \\
& \rho^{n-i} e^{-\mu(1-\rho) x(n-i)+\mu x} \frac{\mu^{n} x^{n-1}}{(n-1) !} \\
= & \frac{n !}{(i-1) !(n-i) !} \rho^{r+n-i} \frac{\mu^{n}}{(n-1) !} \sum_{r=0}^{i-1}\left(\begin{array}{c}
i-1 \\
r
\end{array}\right)(-1)^{r} e^{-\mu(1-\rho)^{r}} e^{-i \rho \mu x} x^{n-1} \\
& =C(n, r, i) \sum_{r=0}^{i-1}\left(\begin{array}{c}
i-1 \\
r
\end{array}\right)(-1)^{r} e^{-\mu(1-\rho)^{r}} e^{-i \rho \mu x} x^{n-1}, x>0, \mu>0
\end{aligned}
$$

where

$$
C(n, r, i)=\frac{n !}{(i-1) !(n-i) !} \rho^{r+n-i} \frac{\mu^{n}}{(n-1) !}
$$

We observe that

$$
\sum_{r=0}^{i-1}\left(\begin{array}{c}
i-1 \\
r
\end{array}\right)(-1)^{r} \rho^{r} e^{-\mu(1-\rho)^{r}}=\left[1-\rho e^{-\mu(1-\rho) x}\right]^{i-1}
$$

The cdf of $i^{\text {th }}$ order statistics $X_{i: n}, 1<i<n$ for waiting time distribution is obtained as

$$
\begin{aligned}
& \Phi_{i: n w}(x)=C(n, r, i) \sum_{r=0}^{i-1}\left(\begin{array}{c}
i-1 \\
r
\end{array}\right)(-1)^{r} e^{-\mu(1-\rho)^{r}} \int_{0}^{x} e^{-i \rho \mu x} x^{n-1} d x \\
& =C(n, r, i) \sum_{r=0}^{i-1}\left(\begin{array}{c}
i-1 \\
r
\end{array}\right)(-1)^{r} e^{-\mu(1-\rho)^{r}} \sum_{j=o}^{\infty} \frac{(-i \rho \mu)^{j}}{j !} \frac{x^{n}}{n}, x>0, \mu>0
\end{aligned}
$$

3.2. Derivation of extreme order statistics. The probability density functions of smallest and largest order statistics can be obtained from equation (3.4) by putting $\mathrm{i}=$ 1 and $\mathrm{i}=\mathrm{n}$ respectively. The probability density functions of smallest and largest order statistics for waiting time distribution are obtained as

$$
\phi_{1: n_{w}}(x)=n e^{-\mu(1-\rho)^{r}} e^{-\rho \mu x} x^{n-1}, x>0, \mu>0
$$

and

$$
\phi_{n: n_{w}}(x)=n \sum_{r=0}^{n-1}\left(\begin{array}{c}
n-1 \\
r
\end{array}\right)(-1)^{r} e^{-\mu(1-\rho)^{r}} e^{-n \rho \mu x} x^{n-1}, x>0, \mu>0
$$

respectively.

The cumulative density functions of smallest and largest order statistics for waiting time 
distribution can be obtained using expression (2.4) and (2.5) as

$$
\begin{aligned}
& \Phi_{1: n w}(x)=n e^{-\mu(1-\rho)^{r}} e^{-\rho \mu x} x^{n-1}, 0<x<\infty \\
& \Phi_{n: n w}(x)=1-n \sum_{r=0}^{n}\left(\begin{array}{l}
n \\
r
\end{array}\right)(-1)^{r} e^{-\mu(1-\rho)^{r}} e^{-n \rho \mu x} x^{n-1}, 0<x<\infty
\end{aligned}
$$

3.3. Moments and recurrence relations of $i^{\text {th }}$ order statistics for waiting time distribution. Let $X_{(1)}, X_{(2)}, \ldots, X_{(n)}$ be independent and identically distributed random sample of size $n$ from a continuous distribution with probability density function $\phi_{X}(x)$ and a cumulative density function $\Phi_{X}(x)$ from Waiting time distribution. From the probability density function of $i^{t h}$ order statistics for waiting time distribution the $p^{t h}$ order moment can be written as

$$
\begin{aligned}
\mu_{p}^{\prime}(i: n)= & \frac{n !}{(i-1) !(n-i) !} \frac{\mu^{n}}{(n-1) !} \int_{0}^{\infty} x^{n+p-1}\left[1-\rho e^{-\mu(1-\rho) x}\right]^{i-1} \\
& {\left[1-\left(1-\rho e^{-\mu(1-\rho) x}\right)\right]^{n-i} e^{-\mu x} d x } \\
= & C(n, i) \int_{0}^{\infty} x^{n+p-1}\left[1-\rho e^{-\mu(1-\rho) x}\right]^{i-1} \\
& {\left[1-\left(1-\rho e^{-\mu(1-\rho) x}\right)\right]^{n-i} e^{-\mu x} d x }
\end{aligned}
$$

Here we applied the binomial expression in (3.9), we get

$$
\begin{aligned}
\mu_{p}^{\prime}(i: n) & =C(n, r, i) \sum_{r=0}^{i-1}\left(\begin{array}{c}
i-1 \\
r
\end{array}\right)(-1)^{r} e^{-\mu(1-\rho)^{r}} \int_{0}^{\infty} e^{-i \rho \mu x} x^{n+p-1} d x \\
& =C(n, r, i) \sum_{r=0}^{i-1}\left(\begin{array}{c}
i-1 \\
r
\end{array}\right)(-1)^{r} e^{-\mu(1-\rho)^{r}} \frac{\gamma(n+p)}{(i \mu \rho)^{n+p}}
\end{aligned}
$$

putting $i=n$, we get the highest order moment which is given by

$$
\mu_{p}^{\prime}(n: n)=\sum_{r=0}^{n-1}\left(\begin{array}{c}
n-1 \\
r
\end{array}\right)(-1)^{r} e^{-\mu(1-\rho)^{r}} \frac{\gamma(n+p)}{(n \mu \rho)^{n+p}}
$$

The recurrence relation for moments of $i^{\text {th }}$ order statistics is given by

$$
\begin{aligned}
\mu_{p+1}^{\prime}(i: n) & =\sum_{r=0}^{n-1}\left(\begin{array}{c}
n-1 \\
r
\end{array}\right)(-1)^{r} e^{-\mu(1-\rho)^{r}} \frac{\gamma(n+p+1)}{(n \mu \rho)^{n+p+1}} \\
\Longrightarrow i \mu \rho \mu_{p+1}{ }^{\prime}(i: n) & =\mu_{p}{ }^{\prime}(i: n)
\end{aligned}
$$

3.4. Mode for waiting time distribution. The modal value equation of the $i^{\text {th }}$ order statistics is

$$
\begin{aligned}
C(n, r, i) \sum_{r=0}^{i-1}\left(\begin{array}{c}
i-1 \\
r
\end{array}\right)(-1)^{r} e^{-\mu(1-\rho)^{r}} \frac{\delta}{\delta x}\left[e^{-i \rho \mu x} x^{n-1}\right] & =0 \\
\Longrightarrow C(n, r, i) \sum_{r=0}^{i-1}\left(\begin{array}{c}
i-1 \\
r
\end{array}\right)(-1)^{r} e^{-\mu(1-\rho)^{r}} e^{-i \rho \mu x} x^{n-2}(n-1-i \rho \mu) & =0
\end{aligned}
$$

3.5. Joint distribution of two order statistics for waiting time distribution. Let $X_{(1)}, X_{(2)}, \ldots, X_{(n)}$ denote the order statistics of a random sample of size $n$ from a continuous distribution with probability density function $\phi_{X}(x)$ and a cumulative density function $\Phi_{X}(x)$. Let us assume that $u=X_{i: n}$ and $v=X_{i: n}$ as $i^{\text {th }}$ and $j^{\text {th }}$ order statistics, 
$(1<i<j<n)$ from a random sample of size $n$, each with probability density function $\phi_{X}(x)$. The joint density function of $u=X_{i: n}$ and $v=X_{i: n}$ is as follows

$$
\begin{aligned}
\phi_{w}(u, v ; n)= & \frac{n !}{(i-1) !(j-i-1) !(n-j) !}\left[\Phi\left(x_{r}\right)\right]^{r-1}\left[\Phi\left(x_{s}\right)-\Phi\left(x_{r}\right)\right]^{j-i-1} \\
& {\left[1-\Phi\left(x_{s}\right)\right]^{n-j} \phi\left(x_{r}\right) \phi\left(x_{s}\right) } \\
= & \frac{n !}{(i-1) !(j-i-1) !(n-j) !}\left[1-\rho e^{-\mu(1-\rho) u}\right]^{i-1} \\
& {\left[\rho e^{-\mu(1-\rho) u}-\rho e^{-\mu(1-\rho) v}\right]^{j-i-1}\left[\rho e^{-\mu(1-\rho) v}\right]^{n-j} } \\
& e^{-\mu u} \frac{\mu^{n} u^{n-1}}{(n-1) !} e^{-\mu v} \frac{\mu^{n} v^{n-1}}{(n-1) !}
\end{aligned}
$$

Using Binomial expansion on (3.11), we get

$$
\begin{aligned}
= & C^{\prime}(i, j ; n) \gamma^{2} \sum_{\alpha=0}^{j-i-1} \sum_{\beta=0}^{n-j}\left(\begin{array}{c}
j-i-1 \\
\alpha
\end{array}\right)\left(\begin{array}{c}
n-j \\
\beta
\end{array}\right)(-1)^{\alpha+\beta}\left(\rho e^{-\mu(1-\rho) v}\right)^{j-i-1+\alpha+\beta} \\
& {\left[\rho e^{-\mu(1-\rho) u}\right]^{\alpha+i-1} e^{-\mu(u+v)} u^{n-1} v^{n-1}, u, v>0, \mu>0 }
\end{aligned}
$$

where

$$
C^{\prime}(i, j ; n)=\frac{n !}{(i-1) !(j-i-1) !(n-j) !} \text { and } \gamma=\frac{\mu^{n}}{(n-1) !}
$$

\section{Derivation of distribution of sample range for waiting time dis- tribution}

Let the sample range of the waiting time distribution be defined as

$$
R=X_{(n)}-X_{(1)}
$$

Also, let

$$
\begin{gathered}
X_{(n)}=x \text { and } X_{(1)}=y \Rightarrow u=x \text { and } \Rightarrow v=y-u \Rightarrow y=u+v \\
J=\left|\begin{array}{ll}
\frac{\delta x}{\delta u} & \frac{\delta x}{\delta v} \\
\frac{\delta y}{\delta u} & \frac{\delta y}{\delta v}
\end{array}\right|=\left|\begin{array}{ll}
1 & 0 \\
1 & 1
\end{array}\right|=1
\end{gathered}
$$

Now using the joint distribution of order statistics

$$
\begin{aligned}
g(u, v)= & \frac{n !}{(1-1) !(n-1-1) !(n-n) !} \Phi^{1-1}[\Phi(u+v)-\Phi(u)]^{n-2} \\
& {[1-\Phi(u+v)]^{n-n} \phi(u) \phi(u+v) } \\
= & \frac{n !}{(n-2) !}[\Phi(u+v)-\Phi(u)]^{n-2} \phi(u) \phi(u+v)
\end{aligned}
$$




$$
\begin{aligned}
g_{1}(v)= & \int g(u, v) d u \\
= & \left.\int_{-\infty}^{\infty} n(n-1) \Phi(u+v)-\Phi(u)\right]^{n-2} \phi(u) \phi(u+v) \\
= & \int_{0}^{\infty} n(n-1)\left[\rho\left[1-e^{-\mu(1-\rho)(u+v)}\right]-\rho\left[1-e^{-\mu(1-\rho) v}\right]\right]^{n-2} d u \\
& \mu \rho(1-\rho) e^{-\mu(1-\rho) u} \mu \rho(1-\rho) e^{-\mu(1-\rho)(u+v)} \\
= & \int_{0}^{\infty} n(n-1)\left[\rho\left[e^{-\mu(1-\rho) u}-e^{-\mu(1-\rho)(u+v)}\right]\right]^{n-2} \\
& \mu \rho(1-\rho) e^{-\mu(1-\rho)(2 u+v)} d u \\
= & \int_{0}^{\infty} n(n-1)\left[e^{-\mu(1-\rho) u}\right]^{n-2}\left[\rho\left[1-e^{-\mu(1-\rho v)}\right]\right]^{n-2} \\
& \mu \rho(1-\rho) e^{-\mu(1-\rho)(2 u+v)} d u \\
= & n(n-1) \mu \rho(1-\rho) e^{-\mu(1-\rho) v}\left[\rho\left[1-e^{-\mu(1-\rho v)}\right]\right]^{n-2} \\
& \int_{0}^{\infty}\left[e^{-\mu(1-\rho) n u}\right] d u \\
= & n(n-1) \mu \rho(1-\rho) e^{-\mu(1-\rho) v}\left[\rho\left[1-e^{-\mu(1-\rho v)}\right]\right]^{n-2} \\
& \left|\frac{e^{-\mu(1-\rho) n u}}{-\mu(1-\rho) n}\right|_{0}^{\infty} d u \\
& \rho^{n}(n-1) \rho(1-\rho) e^{-\mu(1-\rho) v}\left[\rho\left[1-e^{-\mu(1-\rho v)}\right]\right]^{n-2}
\end{aligned}
$$

Therefore the range of the distribution of the waiting time is

$$
\begin{aligned}
P(R=v)= & \rho^{n}(n-1) \rho(1-\rho) e^{-\mu(1-\rho) v} \\
& {\left[\rho\left[1-e^{-\mu(1-\rho v)}\right]\right]^{n-2},-\infty<v<\infty }
\end{aligned}
$$

4.1. Theorem. Let $X_{(1)}, X_{(2)}, \ldots, X_{(n)}$ denote the order statistics of a random sample of size $n$ from a continuous distribution with probability density function $\phi_{X}(x)$ and a cumulative density function $\Phi_{X}(x)$. Then the probability density function of the range of the waiting time distribution in the system is

$$
\begin{aligned}
P(R=s)= & (n-1) \rho(1-\rho) e^{-\mu(1-\rho) v} \\
& {\left[\rho\left[1-e^{-\mu(1-\rho v)}\right]\right]^{n-2},-\infty<v<\infty }
\end{aligned}
$$

Proof. As stated as same in equation (4.1).

\section{Derivation of response time distribution}

Percentiles are frequently used as indicators of performance in both the public and private hospitals. Percentiles provide information about how a patient or thing relates to a larger group. Relative measures of this type are often extremely valuable to researchers employing statistical techniques.

The formula of the mean response time is given by 
Mean number in the system $=$ Arrival rate $\times$ mean response time i.e.,

$$
\begin{aligned}
E(n) & =\lambda E(r) \\
\Longrightarrow F(r) & =\frac{E(n)}{\lambda}=\left(\frac{\rho}{1-\rho}\right) \frac{1}{\lambda}=\frac{\frac{1}{\mu}}{1-\rho}
\end{aligned}
$$

The cumulative distribution function of the response time is given as

$$
F(r)=1-e^{-r \mu(1-\rho)}
$$

The response time is exponentially distributed and $q^{t h}$ percentile is

$$
\begin{aligned}
F(r) & =\frac{q}{100} \Longrightarrow 1-e^{-r \mu(1-\rho)}=\frac{q}{100} \\
\Longrightarrow r_{q} & =\frac{1}{\mu(1-\rho)} \ln \left(\frac{100}{100-q}\right)
\end{aligned}
$$

The cumulative distribution function of the waiting time is

$$
F(w)=1-\rho e^{-w \mu(1-\rho)}
$$

This is a truncated exponential distribution and its $q^{\text {th }}$ percentile is given by

$$
w_{q}=\frac{1}{\mu(1-\rho)} \ln \left(\frac{100 \rho}{100-q}\right)
$$

The above formula is applied only if $q$ is greater than $100(1-\rho)$ and all lower percentiles are zero.

$$
w_{q}=\max \left\{0, \frac{E(w)}{\rho} \ln \left(\frac{100 \rho}{100-q}\right)\right\}
$$

\section{Distribution of sample median}

When the sample size is odd, then the probability density function of the sample median is given by

$$
\begin{aligned}
\phi(x) & =\frac{(2 n+1)}{m !^{2}}[\Phi(z)]^{n}[1-\Phi(z)]^{n} \phi(z) \\
& =\frac{(2 n+1)}{n !^{2}}\left[1-\rho e^{-\mu(1-\rho) z}\right]^{n}\left[\rho e^{-\mu(1-\rho) z}\right]^{n} e^{-\mu z} \frac{\mu^{n} z^{n-1}}{(n-1) !} \\
& =C(n, \gamma) \sum_{s=0}^{r}(-1)^{s} e^{-\mu(s+r)} z^{s+r-1}
\end{aligned}
$$

When the sample size is even, then the probability density function of the sample median is as follows

$$
\begin{aligned}
\phi(x, y) & =\frac{(2 n)}{(n-1) !}[\Phi(x)]^{n-1}[1-\Phi(y)]^{n-1} \phi(x) \phi(y) \\
& =\frac{(2 n)}{(n-1) !}\left[1-\rho e^{-\mu(1-\rho) x}\right]^{n-1}\left[\rho e^{-\mu(1-\rho) y}\right]^{n-1} e^{-\mu x} \frac{\mu^{n} x^{n-1}}{(n-1) !} e^{-\mu y} \frac{\mu^{n} y^{n-1}}{(n-1) !} \\
& =\frac{(2 n)}{(n-1) !}\left[1-\rho e^{-\mu(1-\rho) x}\right]^{n-1}\left[\rho e^{-\mu(1-\rho) y}\right]^{n-1} e^{-\mu(x+y)} \frac{\mu^{n} x^{n-1}}{(n-1) !} \frac{\mu^{n} y^{n-1}}{(n-1) !} \\
& =C(n, \gamma) \sum_{s=0}^{r}(-1)^{s} e^{-\mu(x+y)}(x y)^{n-1}
\end{aligned}
$$




\section{Results}

Here we evaluate the minimum and maximum waiting time of the patients who are in the queue. The table below gives the minimum number of patients in the system and queue for given number of servers during each interval and it is clear that for both the queue and the system, the waiting time drops measurably from $1^{\text {st }}$ to $5^{\text {th }}$ server, after which the drop is trivial. Hence it is concluded that the maximum and minimum number of patients decrease gradually with the increasing number of servers.

Table 1. The maximum and minimum number of patients served and waiting in the system

\begin{tabular}{cccc}
\hline Server & $X_{n}(W)$ & $X_{1}\left(W_{q}\right)$ & $R$ \\
\hline 1 & 5 & 4.16667 & 0.83333 \\
2 & 2.72727 & 2.27273 & 0.1388 \\
3 & 1.82817 & 1.37363 & 0.023148 \\
4 & 1.37125 & 0.931446 & 0.003858 \\
5 & 1.09697 & 0.671899 & 0.000643 \\
6 & 0.914135 & 0.168631 & 0.0001071 \\
7 & 0.783545 & 0.108038 & $2.97687 \mathrm{E}-6$ \\
8 & 0.685602 & 0.0704791 & $4.96145 \mathrm{E}-7$ \\
9 & 0.609424 & 0.0465906 & $8.26908 \mathrm{E}-8$ \\
10 & 0.548481 & 0.0311082 & $1.37818 \mathrm{E}-8$ \\
\hline
\end{tabular}

The percentage points of response and waiting times in the system have been presented in Table(2), Table(3), Table(4) and Table(5). From the tables of percentage points of response time in the system, it is clear that for fixed values of $p$ ( $p=$ $0.25,0.50,0.75,0.90,0.95,0.99$ ) and $\mu$. Percentage points remain same as $\mu$ increases. On the other hand percentage points decreases as $\mu$ increases. Further, the range of smallest and largest order statistics of waiting time distribution and has been presented in Table(1). For chosen values of the parameters and $n$ different values of $r_{q}$ have been obtained for different significant levels. 
Table 2. This table contains percentage of response time in the system when $\mu=1$

\begin{tabular}{ccccccc}
\hline & \multicolumn{5}{c}{$\mathbf{q}$} \\
\cline { 2 - 7 }$\lambda$ & 0.25 & 0.50 & 0.75 & 0.90 & 0.95 & 0.99 \\
\hline 2 & 0.00250 & 0.00501 & 0.00753 & 0.00904 & 0.00955 & 0.00995 \\
3 & 0.00125 & 0.00251 & 0.00376 & 0.00452 & 0.00477 & 0.00497 \\
4 & 0.00083 & 0.00167 & 0.00251 & 0.00301 & 0.00318 & 0.00332 \\
5 & 0.00063 & 0.00125 & 0.00188 & 0.00226 & 0.00239 & 0.00249 \\
6 & 0.00050 & 0.00100 & 0.00151 & 0.00181 & 0.00191 & 0.00199 \\
7 & 0.00042 & 0.00084 & 0.00125 & 0.00151 & 0.00159 & 0.00166 \\
8 & 0.00036 & 0.00072 & 0.00108 & 0.00129 & 0.00136 & 0.00142 \\
9 & 0.00031 & 0.00063 & 0.00094 & 0.00113 & 0.00119 & 0.00124 \\
10 & 0.00028 & 0.00056 & 0.00084 & 0.00100 & 0.00106 & 0.00111 \\
11 & 0.00025 & 0.00050 & 0.00075 & 0.00090 & 0.00095 & 0.00099 \\
12 & 0.00023 & 0.00046 & 0.00068 & 0.00082 & 0.00087 & 0.00090 \\
13 & 0.00021 & 0.00042 & 0.0006 & 30.00075 & 0.00080 & 0.00083 \\
14 & 0.00019 & 0.00039 & 0.00058 & 0.00070 & 0.00073 & 0.00077 \\
15 & 0.00018 & 0.00036 & 0.00054 & 0.00065 & 0.00068 & 0.00071 \\
16 & 0.00017 & 0.00033 & 0.00050 & 0.00060 & 0.00064 & 0.00066 \\
17 & 0.00016 & 0.00031 & 0.00047 & 0.00057 & 0.00060 & 0.00062 \\
18 & 0.00015 & 0.00029 & 0.00044 & 0.00053 & 0.00056 & 0.00059 \\
19 & 0.00014 & 0.00028 & 0.00042 & 0.00050 & 0.00053 & 0.00055 \\
20 & 0.00013 & 0.00026 & 0.00040 & 0.00048 & 0.00050 & 0.00052 \\
\hline
\end{tabular}

Table 3. This table contains percentage of response time in the system when $\mu=2$

\begin{tabular}{ccccccc}
\hline & \multicolumn{5}{c}{$\mathbf{q}$} \\
\cline { 2 - 7 }$\lambda$ & 0.25 & 0.50 & 0.75 & 0.90 & 0.95 & 0.99 \\
\hline 3 & 0.00250 & 0.00501 & 0.00753 & 0.00904 & 0.00955 & 0.00995 \\
4 & 0.00125 & 0.00251 & 0.00376 & 0.00452 & 0.00477 & 0.00497 \\
5 & 0.00083 & 0.00167 & 0.00251 & 0.00301 & 0.00318 & 0.00332 \\
6 & 0.00063 & 0.00125 & 0.00188 & 0.00226 & 0.00239 & 0.00249 \\
7 & 0.00050 & 0.00100 & 0.00151 & 0.00181 & 0.00191 & 0.00199 \\
8 & 0.00042 & 0.00084 & 0.00125 & 0.00151 & 0.00159 & 0.00166 \\
9 & 0.00036 & 0.00072 & 0.00108 & 0.00129 & 0.00136 & 0.00142 \\
10 & 0.00031 & 0.00063 & 0.00094 & 0.00113 & 0.00119 & 0.00124 \\
11 & 0.00028 & 0.00056 & 0.00084 & 0.00100 & 0.00106 & 0.00111 \\
12 & 0.00025 & 0.00050 & 0.00075 & 0.00090 & 0.00095 & 0.00099 \\
13 & 0.00023 & 0.00046 & 0.00068 & 0.00082 & 0.00087 & 0.00090 \\
14 & 0.00021 & 0.00042 & 0.00063 & 0.00075 & 0.00080 & 0.00083 \\
15 & 0.00019 & 0.00039 & 0.00058 & 0.00070 & 0.00073 & 0.00077 \\
16 & 0.00018 & 0.00036 & 0.00054 & 0.00065 & 0.00068 & 0.00071 \\
17 & 0.00017 & 0.00033 & 0.00050 & 0.00060 & 0.00064 & 0.00066 \\
18 & 0.00016 & 0.00031 & 0.00047 & 0.00057 & 0.00060 & 0.00062 \\
19 & 0.00015 & 0.00029 & 0.00044 & 0.00053 & 0.00056 & 0.00059 \\
20 & 0.00014 & 0.00028 & 0.00042 & 0.00050 & 0.00053 & 0.00055 \\
21 & 0.00013 & 0.00026 & 0.00040 & 0.00048 & 0.00050 & 0.00052 \\
\hline
\end{tabular}


Table 4. This table contains percentage of waiting time in the system when $\mu=1$

\begin{tabular}{ccccccc}
\hline & \multicolumn{5}{c}{$\mathbf{q}$} \\
\cline { 2 - 7 }$\lambda$ & 0.25 & 0.50 & 0.75 & 0.90 & 0.95 & 0.99 \\
\hline 2 & 0.690644 & 0.688135 & 0.685619 & 0.684106 & 0.683602 & 0.683198 \\
3 & 0.548055 & 0.5468 & 0.545542 & 0.544786 & 0.544533 & 0.544331 \\
4 & 0.461264 & 0.460427 & 0.459589 & 0.459085 & 0.458916 & 0.458782 \\
5 & 0.401734 & 0.401106 & 0.400477 & 0.400099 & 0.399973 & 0.399872 \\
6 & 0.357851 & 0.357349 & 0.356846 & 0.356544 & 0.356443 & 0.356362 \\
7 & 0.323901 & 0.323483 & 0.323064 & 0.322812 & 0.322727 & 0.32266 \\
8 & 0.296705 & 0.296347 & 0.295988 & 0.295772 & 0.295699 & 0.295642 \\
9 & 0.27434 & 0.274027 & 0.273712 & 0.273523 & 0.27346 & 0.273409 \\
10 & 0.255565 & 0.255286 & 0.255006 & 0.254838 & 0.254782 & 0.254737 \\
11 & 0.239539 & 0.239288 & 0.239037 & 0.238885 & 0.238835 & 0.238795 \\
12 & 0.225673 & 0.225445 & 0.225216 & 0.225079 & 0.225033 & 0.224996 \\
13 & 0.213537 & 0.213328 & 0.213118 & 0.212992 & 0.21295 & 0.212917 \\
14 & 0.202812 & 0.202619 & 0.202425 & 0.202309 & 0.20227 & 0.202239 \\
15 & 0.193253 & 0.193074 & 0.192894 & 0.192786 & 0.19275 & 0.192721 \\
16 & 0.184672 & 0.184505 & 0.184337 & 0.184237 & 0.184203 & 0.184176 \\
17 & 0.176919 & 0.176763 & 0.176605 & 0.176511 & 0.176479 & 0.176454 \\
18 & 0.169875 & 0.169727 & 0.169579 & 0.16949 & 0.16946 & 0.169437 \\
19 & 0.163441 & 0.163301 & 0.163162 & 0.163078 & 0.16305 & 0.163027 \\
20 & 0.157538 & 0.157406 & 0.157274 & 0.157194 & 0.157168 & 0.157146 \\
\hline
\end{tabular}

Table 5. This table contains percentage of waiting time in the system when $\mu=2$

\begin{tabular}{ccccccc}
\hline & \multicolumn{5}{c}{$\mathbf{q}$} \\
\cline { 2 - 7 }$\lambda$ & 0.25 & 0.50 & 0.75 & 0.90 & 0.95 & 0.99 \\
\hline 3 & 0.40296198 & 0.40045257 & 0.39793684 & 0.39642436 & 0.3959197 & 0.39551578 \\
4 & 0.34532203 & 0.34406732 & 0.34280946 & 0.34205322 & 0.34180088 & 0.34159892 \\
5 & 0.30459587 & 0.3037594 & 0.30292082 & 0.30241666 & 0.30224844 & 0.3021138 \\
6 & 0.27402729 & 0.27339994 & 0.27277101 & 0.27239289 & 0.27226672 & 0.27216574 \\
7 & 0.25005197 & 0.24955009 & 0.24904694 & 0.24874444 & 0.24864351 & 0.24856273 \\
8 & 0.23063187 & 0.23021364 & 0.22979435 & 0.22954227 & 0.22945816 & 0.22939084 \\
9 & 0.21451061 & 0.21415212 & 0.21379273 & 0.21357666 & 0.21350457 & 0.21344687 \\
10 & 0.20086685 & 0.20055317 & 0.20023871 & 0.20004965 & 0.19998656 & 0.19993607 \\
11 & 0.18913833 & 0.18885951 & 0.18857998 & 0.18841193 & 0.18835585 & 0.18831097 \\
12 & 0.17892563 & 0.17867469 & 0.17842312 & 0.17827187 & 0.17822141 & 0.17818101 \\
13 & 0.16993628 & 0.16970815 & 0.16947945 & 0.16934195 & 0.16929607 & 0.16925935 \\
14 & 0.16195058 & 0.16174147 & 0.16153182 & 0.16140578 & 0.16136373 & 0.16133007 \\
15 & 0.15479999 & 0.15460696 & 0.15441344 & 0.1542971 & 0.15425828 & 0.15422721 \\
16 & 0.14835274 & 0.1481735 & 0.14799381 & 0.14788577 & 0.14784972 & 0.14782087 \\
17 & 0.1425042 & 0.14233691 & 0.14216919 & 0.14206836 & 0.14203472 & 0.14200779 \\
18 & 0.13717009 & 0.13701325 & 0.13685602 & 0.13676149 & 0.13672995 & 0.1367047 \\
19 & 0.13228169 & 0.13213407 & 0.13198609 & 0.13189712 & 0.13186743 & 0.13184367 \\
20 & 0.12778233 & 0.12764292 & 0.12750316 & 0.12741913 & 0.12739109 & 0.12736865 \\
21 & 0.12362485 & 0.12349277 & 0.12336037 & 0.12328076 & 0.1232542 & 0.12323294 \\
\hline
\end{tabular}


Table 6. This table contains percentage of waiting time in the system when $\mu=3$

\begin{tabular}{ccccccc}
\hline & \multicolumn{5}{c}{$\mathbf{q}$} \\
\cline { 2 - 7 }$\lambda$ & 0.25 & 0.50 & 0.75 & 0.90 & 0.95 & 0.99 \\
\hline 4 & 0.28517894 & 0.28266953 & 0.28015381 & 0.27864133 & 0.27813666 & 0.27773274 \\
5 & 0.25416125 & 0.25290654 & 0.25164868 & 0.25089244 & 0.25064011 & 0.25043815 \\
6 & 0.23021468 & 0.22937821 & 0.22853964 & 0.22803548 & 0.22786726 & 0.22773262 \\
7 & 0.21119868 & 0.21057133 & 0.2099424 & 0.20956428 & 0.20943811 & 0.20933713 \\
8 & 0.19566522 & 0.19516334 & 0.1946602 & 0.1943577 & 0.19425677 & 0.19417598 \\
9 & 0.18268486 & 0.18226662 & 0.18184734 & 0.18159526 & 0.18151115 & 0.18144383 \\
10 & 0.17163852 & 0.17128004 & 0.17092065 & 0.17070458 & 0.17063248 & 0.17057478 \\
11 & 0.16209748 & 0.16178381 & 0.16146934 & 0.16128028 & 0.1612172 & 0.16116671 \\
12 & 0.15375458 & 0.15347576 & 0.15319623 & 0.15302818 & 0.15297211 & 0.15292723 \\
13 & 0.14638339 & 0.14613245 & 0.14588088 & 0.14572963 & 0.14567917 & 0.14563877 \\
14 & 0.1398129 & 0.13958477 & 0.13935607 & 0.13921857 & 0.13917269 & 0.13913597 \\
15 & 0.13391123 & 0.13370211 & 0.13349247 & 0.13336643 & 0.13332437 & 0.13329072 \\
17 & 0.12857487 & 0.12838184 & 0.12818832 & 0.12807198 & 0.12803316 & 0.12800208 \\
18 & 0.12372128 & 0.12354204 & 0.12336234 & 0.12325431 & 0.12321826 & 0.12318941 \\
19 & 0.11928376 & 0.11911646 & 0.11894875 & 0.11884791 & 0.11881427 & 0.11878734 \\
20 & 0.11520772 & 0.11505088 & 0.11489365 & 0.11479912 & 0.11476758 & 0.11474233 \\
21 & 0.11144805 & 0.11130044 & 0.11115245 & 0.11106348 & 0.1110338 & 0.11101004 \\
22 & 0.10796706 & 0.10782764 & 0.10768788 & 0.10760386 & 0.10757582 & 0.10755338 \\
\hline
\end{tabular}

\section{Conclusion}

The obtained results show that the expected value of the maximum and minimum number of patients decreases gradually with the increasing number of servers. Besides, it is mentioned that the system will be almost empty after the $10^{\text {th }}$ server. That is, the patient will get the service as soon as she or he arrive and will leave the system before the next arrival. This solution accords with the fact that the service rate is greater than the arrival rate. The range of the waiting time $R$ decreases gradually to zero as the number of servers increases.

\section{Acknowledgement}

We are sincerely thankful to UGC-BSR Scheme, Government of India for granting us the financial assistance to carry out this research work.

\section{References}

[1] Aleem, M. Contributions to the theory of order statistics and selection procedures for restricted families of probability distribution. $\mathrm{PhD}$ thesis, Bahauddin Zakariya University, Multan, 1998.

[2] Arnold, B., Balakrishnan, N., and Nagaraja, H. A first course in order statistics. wiley, New York, 1992.

[3] Artalejo, J. R., Economou, A., and Lopez-Herrero, M. J. (2007). Algorithmic analysis of the maximum queue length in a busy period for the $M / M / c$ retrial queue. INFORMS Journal on Computing, 19(1), 121-126, 2007.

[4] Asmussen, S. (1998). Extreme value theory for queues via cycle maxima. Extremes, 1(2), 137-168, 1998.

[5] Bagui, S. C. CRC Handbook of Percentiles of Non-central T-distributions. CRC Press, 1993.

[6] Bhat, U. N. An introduction to queueing theory: modeling and analysis in applications. Birkhauser, 2015. 
[7] David, H. and Nagaraja, H. Order Statistics. Wiley Series in Probability and Statistics. Wiley, 2004

[8] Nadarajah, S. and Pal, M. Explicit expressions for moments of gamma order statistics. Bulletin of the Brazilian Mathematical Society, New Series, 39(1), 45-60, 2008.

[9] Dhar, S., Das, K. K. and Mahanta, L. B. Comparative study of waiting and service costs of single and multiple server system: A case study on an outpatient department. International Journal of Scientifc Footprints, 3(2), 18-30, 2014.

[10] Shawky, A. and Bakoban, R. Order statistics from exponentiated gamma distribution and associated inference. Int. J. Contemp. Math. Sciences, 4(2), 71-91, 2009.

[11] White, J. S. Tables of normal percentile points. Journal of the American Statistical Association, 65(330),635-638, 1970.

[12] Tarabia, AMK. A new formula for the transient behaviour of a non-empty $M / M / 1 / 8$ queue, Applied mathematics and computation 132 (1), 1-10, 2002.

[13] Mahanta, L. B., Das, K. K., and Dhar, S. A queuing model for dealing with patients with severe disease. Electronic Journal of Applied Statistical Analysis, 9(2):362-370, 2016.

[14] Serfozo, Richard F.Extreme values of birth and death processes and queues,Stochastic Processes and their Applications, 27, 291-306,1987.

[15] Park, Y. S. Asymptotic distributions of maximum queue lengths for $M / G / 1$ and $G I / M / 1$ systems. Journal of the Korean Statistical Society, 24(1), 19-29, 1994.

[16] Dhar, S., Das, K. K. and Mahanta, L. B. An infinite server queueing model with varying arrival and departures rates for health care system. International Journal of Pure and Applied Mathematics 113(5), 583-593, 2017.

[17] Medhi, J. Stochastic models in queueing theory. Academic Press, 2002. 